\title{
The motor neuron disease mouse model hSOD1- G93A presents a non-canonical profile of senescence biomarkers in the spinal cord
}

\author{
Pascual Torres \\ IRBLleida-UdL \\ Carlos Anerillas \\ IRBLleida-UdL \\ Mario Encinas \\ IRBLleida-UdL
}

Monica Povedano

Hospital de Bellvitge

Pol Andrés-Benito

CIBERNED

Isidro Ferrer

CIBERNED

Victòria Ayala

IRBLleida-UdL

Reinald Pamplona

IRBLleida-UdL

Manuel Portero-0tin ( $\nabla$ manuel.portero@mex.udl.cat )

IRBLleida-UdL https://orcid.org/0000-0002-1823-0299

\section{Short report}

Keywords: Amyotrophic lateral sclerosis, navitoclax, senolytic, neuroinflammation, therapy, cell cycle, cryptic exon

Posted Date: February 15th, 2021

DOl: https://doi.org/10.21203/rs.3.rs-104871/v3

License: (c) (1) This work is licensed under a Creative Commons Attribution 4.0 International License. Read Full License 


\section{Abstract}

Recent evidence demonstrates a pathological role for senescent cells in Alzheimer's and Parkinson's diseases. The present study aimed to show senescence mechanisms including senescence-associated secretory phenotype (SASP) in the familial amyotrophic lateral sclerosis (ALS) transgenic mouse model hSOD1-G93A. We evaluated, as senescence biomarkers, the expression of p16 and p21 with reversetranscriptase quantitative PCR (RT-qPCR), immunofluorescence (IF), and immunohistochemistry (IHC), as well as the senescence-associated b galactosidase (SA-b-gal) activity in the lumbar spinal cords (LSC) of this model. As SASP markers, we quantified the mRNA levels of $\| 1 \mathrm{a}$, I6, Ifna, and Ifnb. Furthermore, we explored if an alteration of alternative splicing is associated with senescence phenomena in this model. Thus, we quantified the Adipor2 cryptic exon inclusion levels, a specific splicing variant repressed by TARDNA binding of $43 \mathrm{kDa}$ (TDP-43), using RT-qPCR. Our results show an atypical senescence-profile in LSC from transgenic mice, increasing p16 and p21 mRNA and protein levels in glial cells with a mostly cytoplasmic pattern, without the canonical increase in SA-beta-gal activity in these cells. Consistent with enhanced SASP, there is an increase in II1a and 116 expression. Also, TDP-43 splicing activity is compromised in this ALS model, in a direct relationship with the increase in p16 expression. However, senolytic drug Navitoclax -with reported benefits in Alzheimer and Parkinson disease mouse models does not alter the present model's disease progression. Navitoclax neither eliminates cells expressing senescence and nor represses the expression of SASP related genes. Globally, our findings support the existence of a non-canonical profile of senescence biomarkers in the LSC of the ALS model hSOD1-G93A.

\section{Introduction}

ALS is an age-related, neurodegenerative disease characterized by the loss of motor neurons with an unfavorable outcome ( $<5 \%$ survival at 5 years after diagnosis). Cellular senescence was first described by Hayflick in the 1960s as a limitation on division of normal cells in vitro [1]. The cellular mechanisms behind this phenomenon were later described, with an important role for cell cycle inhibitors, highlighting p16-INK4a as the major contributor. Another hallmark of senescent cells is the increase in $\beta$ galactosidase, commonly known as SA- $\beta$-gal which is associated with an increase in lysosomal biogenesis. Cellular senescence has been described as a barrier against oncogenesis, with a tradeoff where these cells can develop a pro-inflammatory status known as SASP. This process reflects an attempt to induce tissue repair in which senescent cells, usually accumulating DNA damage, can stimulate its clearance by the immune system. Regarding neurodegenerative diseases, several groups have independently demonstrated the presence of senescent glial cells and SASP in the central nervous system (CNS).

Another process related to aging is the change in alternative splicing (AS), a conserved mechanism that increases the complexity of the proteome. TDP-43 regulates a large number of AS events in a complex way. Several evidence support the role of TDP-43 pathology in age-related neurodegenerative processes and physiological aging [2]. Most AS events regulated by TDP-43 involve the repression of a set of nonconserved (cryptic) exons which are abnormally incorporated into mRNA in ALS. In this line, we previously 
quantified the rate of inclusion of cryptic exons in nervous tissue from ALS donors and cellular models and found a positive correlation with age at death [2].

\section{Results And Discussion}

To clarify whether senescence-associated phenomena and TDP-43 dysfunction could be implicated in ALS, we measured the abovementioned variables in the familial ALS transgenic mouse model hSOD1G93A at different disease stages. The senescence markers $p 16$ and $p 21$, typical biomarkers of senescent cells [3], were analyzed in LSC. Using two different technics (IHC and IF), we characterized the cellular expression pattern of $\mathrm{p} 16$. The results show that the expression of $p 16$ mRNA was progressively increased during disease evolution (Fig. 1a), whereas $p 21$ mRNA levels were only higher at the end-stage (Fig. 1b). p16 and p21 exhibited a predominantly cytoplasmic pattern (Fig. 1c). As shown by IF, p16 positive cells were microglia (Iba1+ cells) (Fig. S1a) and astroglia (GFAP+ cells) (Fig. S1b). These results indicate dynamic changes in cellular senescence-associated markers and SASP related to disease evolution. $p 16$ expression is highly expressed before the symptomatology in our transgenic mice. This fact suggests a role for p16 in disease initiation and progression. Interestingly, senescence-associated cell cycle arrest in an early symptomatic stage (120d) is driven exclusively by $p 16$, whereas $p 21$ only increases later in this model. This may be seen as a result of the late-onset activation of p53 and the DNA damage response pathway, similar to what occurs in the senescence process in microglia. In contrast to p21 (related to reversible cell cycle arrest or quiescence), the senescence process depends heavily on prolonged p16 expression. Strictly speaking, our work and most published articles on 'senescence' do not demonstrate an always irreversible cell cycle arrest. There may be divergent processes sharing common biomarkers. This is the case with macrophage polarization, in which p16 expression and SA- $\beta$-gal activity are physiological, reversible, and not associated with cellular senescence [4]. In this line, cytoplasmic p16 can regulate cell migration in a manner similar to cyclin D1. This evidence reflects a convergent pathway of cell cycle- and senescence-associated proteins regulating cytoskeleton functions. In the case of ALS, cytoskeleton regulators like Rac1 and Cdc42 are implicated in the disease progression and neuroinflammation [5]. Thus, we hypothesized that cytoplasmic p16 could have a similar role in ALS. Like p16 cytoplasmic functions, p21 inhibits the ROCK/LIMK/Cofilin Pathway through MAPK signaling, inducing cytoskeleton remodeling.

We also analyzed another senescence canonical biomarker: SA- $\beta$-gal activity. The main cellular populations expressing SA- $\beta$-gal in ventral LSC are the motor neuron cells (Nissl+cells in the ventral horn, with a motor-neuron compatible cellular size). Neurons of other LSC locations and the vast majority of Nissl- do not show SA- $\beta$-gal activity (Fig. 1d and Fig S2). Interestingly, SA- $\beta$-gal activity was reduced during disease progression in motor neurons and in a small fraction of Nissl-cells (compatible with glia). Our findings agree with previously shown data demonstrating that SA- $\beta$-gal activity in neurons is not associated with senescence, although it is increased in aging mouse brain [6]. Our results suggest that motor neurons contain more lysosomes in cell body than other cells, and that their biogenesis is compromised in this ALS mouse model. In this line, lysosomal mass deficit has already described in this 
model, highlighting a role of hSOD1 aggregates disturbing lysosomal biogenesis [7] and potentially explaining our results from the SA- $\beta$-gal activity assay.

Another marker commonly employed in senescence description is the increase in cytokines linked to SASP. In this case, we quantified the expression of typical SASP markers //1a and //6. We analyzed as well the expression of Ifna and Ifnb (corresponding to type-I IFN response) as they are postulated as latesenescence markers and could be helpful in determining senescence progression in the LSC of this model. The expression of Ifna was not detected in any of the analyzed samples (data not shown). We observed a different pattern of expression between I/1a (Fig. 1e) and I/6 (Fig. 1f). I/1a is increased in the pre-symptomatic stage and is known to be the upstream regulator of IL-6 in SASP [8]. IL-6 is increased in cerebrospinal fluid in ALS, Alzheimer's, and Parkinson's disease [9]. In contrast, Ifnb expression (Fig. 1g) is not altered, which could indicate that senescence in this model does not evolve a late phase. Overall, this might reflect a complex interaction between senescence, SASP, and changes in reactive glial cells and neurodegeneration.

Regarding TDP-43 splicing function, in mice it controls the inclusion in Adipor2 mRNA (Fig S3a,b). In line with loss of TDP-43 function in this model, cryptic exon inclusion in Adipor2 mRNA was higher in lumbar spinal cord in end-stage mice (Fig. 1h) and positively correlated with $p 16$ expression (Fig. 1i). The present data are the first to show specific alteration regarding splicing function in this ALS model. Notably, this process is associated with an increase in the senescence marker $p 16$, and the two processes are likely to be linked in the same pathway. Of note, increased $p 16$ seem restricted to central nervous system, as sciatic nerve does not show these changes (Fig. S3c), in contrast with Adipor2 cryptic exon inclusion, which was also increased in sciatic nerve (Fig. S3d). Noteworthy, increased Adipor2 cryptic exon was associated with loss of Adipor 2 mRNA levels, suggesting increased non-sense mediated decay in both locations (Fig. S3e).

We wanted to explore the potential benefits of senolytic treatment due to the higher expression of senescence related genes in this mouse model. We performed Navitoclax treatment following the protocol described for Alzheimer's disease mouse model [10]. The treatment was initiated at 90 days old and finished at end point (Figure S4a). We estimated the disease progression by weight loss. Navitoclax treatment did not prevent weight loss, neither prolonged survival (Figure S4b and 1j). Finally, we quantified senescence and SASP genes in lumbar spinal cord. None of the analyzed genes showed statistically significant differences (Figure S4c). These results suggest differences in molecular effectors between Alzheimer's and ALS.

Navitoclax is an inhibitor of antiapoptotic protein Bcl2. Senescent cells are highly dependent of different antiapoptotic members. Senolysis is achieved when this antiapoptotic protein is inhibited, promoting cell death. Navitoclax treatment is not enough to slow the disease progression and does not extend the survival. In contrast with data in Alzheimer's and Parkinson's disease models, this treatment does not prevent the increase of senescence and SASP markers. It suggests that senescence phenotype is not driven by Bcl2 expression of stressed or aged cells in this model. Further studies are warranted to 
determine whether senescence-linked phenomena are mechanistically involved in this fatal disease, clearing the pathway for therapeutic development.

\section{Conclusions}

The LSC from the hSOD1-G93A mouse, a model of familial ALS, exhibits a non-canonical profile of senescence biomarkers. This profile is characterized by an early increase in $p 16$ and a late increase in $p 21$, with both displaying a mainly cytoplasmic pattern in glial cells without an increase in SA- $\beta$-gal activity. In the case of SASP, it also has a dynamic profile with increasing levels of /17a from the presymptomatic stage onward and an acute peak of expression in end-stage transgenic mice. Regarding AS, this tissue shows a dysfunctional splicing activity of TDP-43 in end-stage ALS mice. This is the first time that senescence markers, SASP, and TDP-43-associated splicing dysfunction have been described in this ALS mouse model.

\section{List Of Abbreviations}

ALS: amyotrophic lateral sclerosis

AS: Alternative splicing

CNS: Central nervous system

IF: Immunofluorescence

IHC: Immunohistochemistry

LSC: Lumbar spinal cord

RT-Qpcr: Reverse-transcriptase quantitative PCR

SASP: Senescence-associate secretory phenotype

SA-b-gal: Senescence-associated beta galactosidase

TDP-43: TAR-dna binding of $43 \mathrm{kDa}$

\section{Declarations}

\section{Ethics approval and consent to participate}

This study was approved by the Animal Research and Ethics Committee at the University of Lleida.

\section{Consent to publication}

Not applicable. 
Availability of data and materials

The datasets used and/or analysed during the current study are available from the corresponding author on reasonable request.

\section{Competing interests}

The authors declare that they have no competing interests.

\section{Funding}

Grants were received from the Instituto de Salud Carlos III (PI 17-000134, PI 20-0155) to MPO, from the Generalitat de Catalunya 2017SGR696 to RP, and from the Ministerio de Ciencia, Innovación y Universidades (BFU2017-83646-P, AEI,FEDER, UE) to ME. PT is a predoctoral fellow from the Spanish Ministry of Education [FPU16/01446]. Support was also received in the form of a FUNDELA Grant, RedELA-Plataforma Investigación and the Fundació Miquel Valls (Jack Van den Hoek donation). FEDER funds are acknowledged ("A way to make Europe"). These funding bodies had no roles in the design of the study and collection, analysis, and interpretation of data and in writing the manuscript.

\section{Author's contributions}

PT, PAB and IF performed IF and IHC assays; PT, CA and ME analyzed and interpreted tissue IHC slides regarding histological scores of inflammation and SA-beta-GAL staining; MPO and RP Planned the experimental approach and performed data analyses; PT, CA and ME planned and performed RT-qPCR analyses. PT, MP and VA drafted the manuscript and analyzed immunohistochemical data. IF and MPO revised and prepared final form of the manuscript. All authors read and approved the final manuscript.

\section{Acknowledgements}

This study was supported by the Scientific and Technical Service of Immunohistochemistry, Lleida Institute for Biomedical Research, Dr. Pifarré Foundation, IRBLleida. We thank T. Yohannan for editorial assistance.

\section{References}

1. Hayflick L, Moorhead PS. The serial cultivation of human diploid cell strains. Exp Cell Res. 1961;25:585-621. doi:10.1016/0014-4827(61)90192-6.

2. McAleese KE, Walker L, Erskine D, Thomas AJ, McKeith IG, Attems J. TDP-43 pathology in Alzheimer's disease, dementia with Lewy bodies and ageing. Brain Pathol. 2017;27:472-479. doi:10.1111/bpa.12424.

3. Coppé J-P, Desprez P-Y, Krtolica A, Campisi J. The senescence-associated secretory phenotype: the dark side of tumor suppression. Annu Rev Pathol. 2010;5:99-118. doi:10.1146/annurev-pathol$121808-102144$. 
4. Hall BM, Balan V, Gleiberman AS, Strom E, Krasnov P, Virtuoso LP, et al. p16(Ink4a) and senescenceassociated $\beta$-galactosidase can be induced in macrophages as part of a reversible response to physiological stimuli. Aging (Albany, NY). 2017;9:1867-1884. doi:10.18632/aging.101268.

5. D'Ambrosi N, Rossi S, Gerbino V, Cozzolino M. Rac1 at the crossroad of actin dynamics and neuroinflammation in Amyotrophic Lateral Sclerosis. Front Cell Neurosci. 2014;8:279. doi:10.3389/fncel.2014.00279.

6. Piechota M, Sunderland P, Wysocka A, Nalberczak M, Sliwinska MA, Radwanska K, et al. Is senescence-associated $\beta$-galactosidase a marker of neuronal senescence? Oncotarget. 2016;7:81099-81109. doi:10.18632/oncotarget.12752.

7. Xie Y, Zhou B, Lin M-Y, Sheng Z-H. Progressive endolysosomal deficits impair autophagic clearance beginning at early asymptomatic stages in fALS mice. Autophagy. 2015;11:1934-1936. doi:10.1080/15548627.2015.1084460.

8. Orjalo AV, Bhaumik D, Gengler BK, Scott GK, Campisi J. Cell surface-bound IL-1alpha is an upstream regulator of the senescence-associated IL-6/IL-8 cytokine network. Proc Natl Acad Sci USA. 2009;106:17031-17036. doi:10.1073/pnas.0905299106.

9. Chen X, Hu Y, Cao Z, Liu Q, Cheng Y. Cerebrospinal Fluid Inflammatory Cytokine Aberrations in Alzheimer's Disease, Parkinson's Disease and Amyotrophic Lateral Sclerosis: A Systematic Review and Meta-Analysis. Front Immunol. 2018;9:2122. doi:10.3389/fimmu.2018.02122.

10. Bussian TJ, Aziz A, Meyer CF, Swenson BL, van Deursen JM, Baker DJ. Clearance of senescent glial cells prevents tau-dependent pathology and cognitive decline. Nature. 2018;562:578-582. doi:10.1038/s41586-018-0543-y.

\section{Figures}



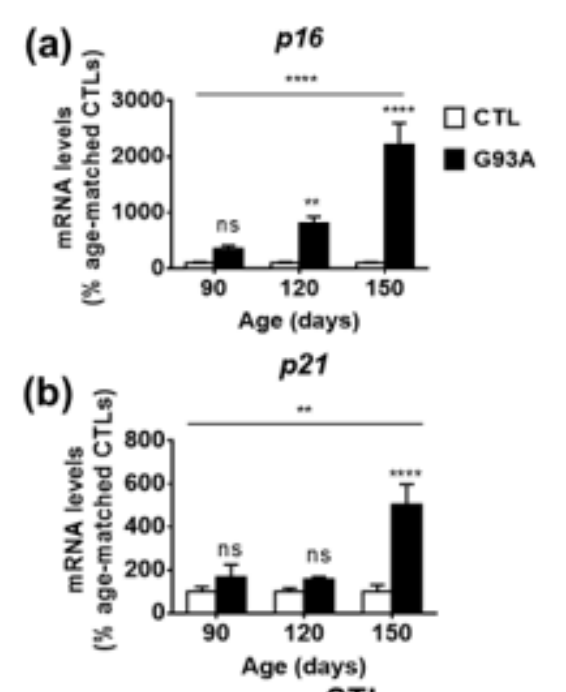

(d)

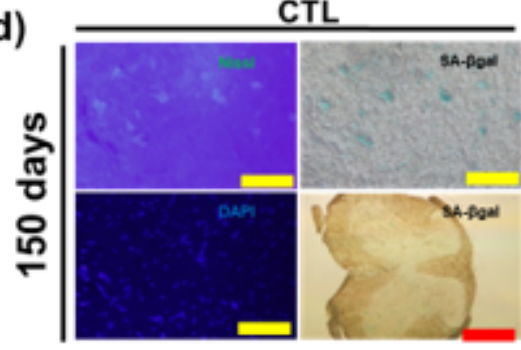

(e)

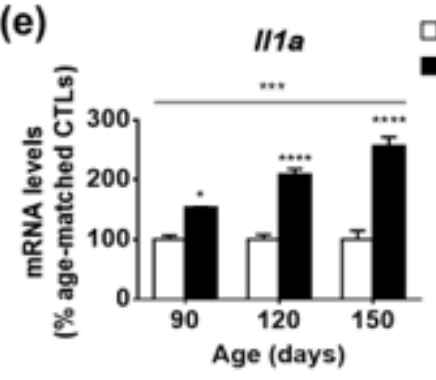

(h)

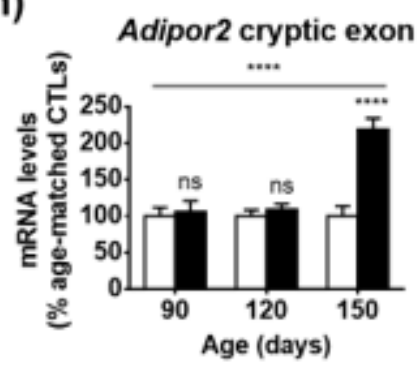

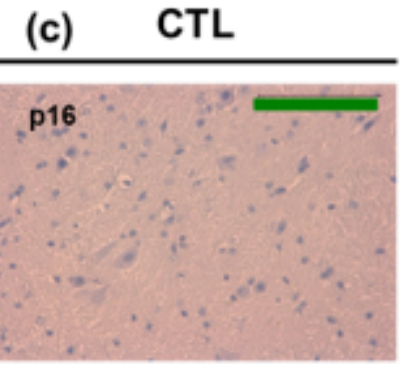

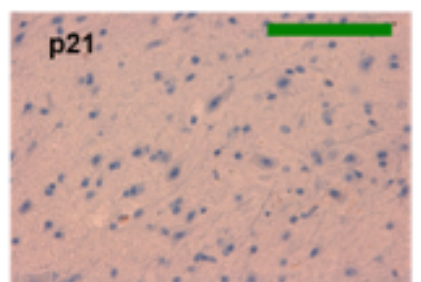

hSOD1-G93A
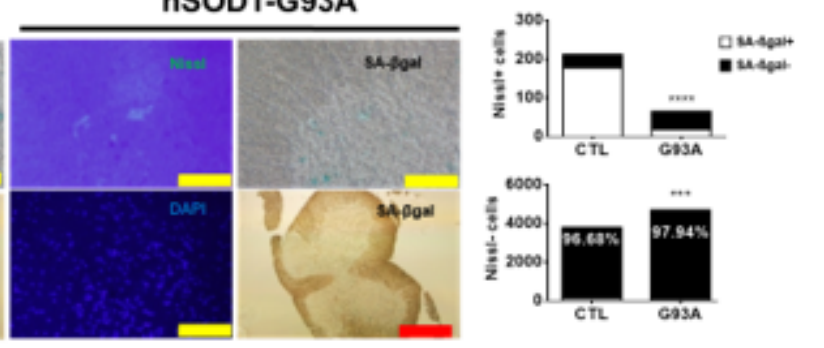

(g)

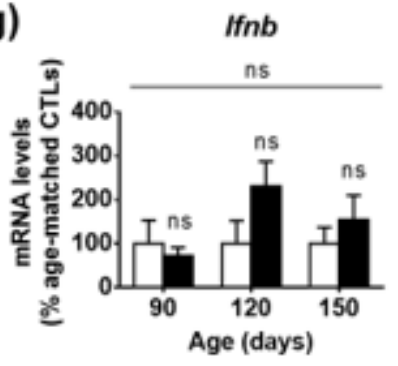

Figure 1

Increase in senescence markers in spinal cord during ALS progression. p16 expression was progressively higher at 120 days and 150 days (a), whereas p21 were only found to be increased at end stage (b). Both p16 and p21 exhibited cytoplasmic staining (c). Nissl+ cells of the ventral horn of the spinal cord (compatible with motor neurons) are the main contributor to SA-beta-gal activity in lumbar spinal cord and are almost depleted in this activity in hSOD1-G93A mice at 150 days, similarly to Nissl- (glia) cells (d). SASP markers and TDP-43 dysfunction are increased in LSC from transgenic hSOD-G93A mice: II1a 
mRNA was higher from pre-symptomatic stage onward in transgenic mice and increased in later stages (e). II6 expression (f) was induced in end-stage hSOD1-G93A mice, in contrast with Ifnb (g). Cryptic exon in Adipor2 mRNA was more incorporated in 150-day-old transgenic mice (h). The inclusion ratio of Adipor2 cryptic exon positively correlated with p16 expression (i) in LSC. Senolytic treatment using Navitoclax does not slow the disease progression, as shown by survival time was also unaltered between groups (j). Values are expressed as mean \pm SEM. Ns indicates $p>0.05$; $* \star p<0.01 ; * \star \star p<0.001$; $* \star \star \star p<<$ 0.0001 for Student's t test or ANOVA, when appropriate. Red arrows indicate 16 and p21 positive cells. Green scale bar represents $100 \mu \mathrm{m}$, yellow scale bar represents $500 \mu \mathrm{m}$, and red scale bar represents $2500 \mu \mathrm{m} . \mathrm{n}=4$ from each genotype and age.

\section{Supplementary Files}

This is a list of supplementary files associated with this preprint. Click to download.

- SUPPLEMENTALMATERIAL.docx 